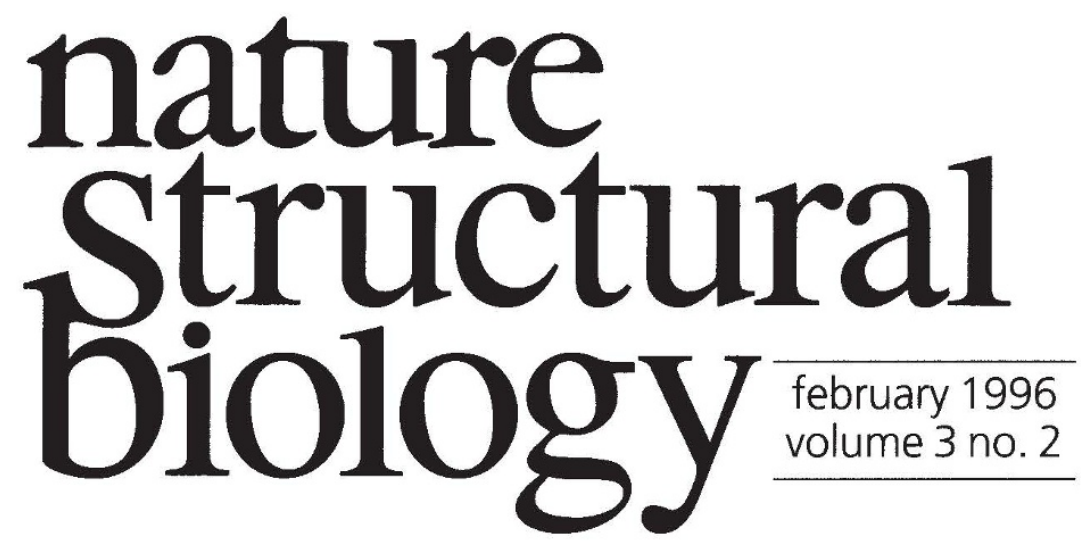

\title{
Information please
}

Structural biologists--perhaps more than adherents to any other biological subdiscipline-rely on computer databases and research tools to keep track of what the community already knows and to organize that information in a meaningful way. One who plunders the trove of known biological structures for enlightening similarities can make as good a living as the one who actually generated the results in the first place. Without computers this task would be difficult, to say the least. Prodigious feats of memory and synthesis were known before the advent of the computer database, particularly in religious traditions: there are still those who memorize the Bhaghavad Gita in multiple dialects; mediaeval European monks also excelled at gathering and cataloguing large amounts of information. Nonetheless, purely human methods are usually too inefficient to deal with the accelerating amounts of information at our disposal, notwithstanding scientists occasionally monastic lifestyles.

Structural biologists depend on community resources, such as structural databases, and particularly those most widely accessible over the internet. Who can imagine sustained progress in this field without the PDB or its equivalent? Besides this database, the community relies on other structural research tools to advance the field. For example, having solved a structure, one may wish to compare it with others, perhaps to find a structurally related protein(s), which may provide evolutionary and functional insight. Many significant structural similarities have been found by eye, but not all structural biologists are so talented (or lucky). Another avenue is collaboration with a group that specializes in structural comparison, using a computer program designed to search structural databases ${ }^{1}$. Fortunately, these groups are generally happy to provide assistance. However, as the number of structures increases, recognizing similarity by eye in a systematic way will become more difficult, and groups specializing in comparison may find themselves overwhelmed by requests for help. Further, given that any individual comparison program has weaknesses, an investigator may well want to test a new structure against several such programs.

One relatively new and ambitious research tool is Entrez (www.ncbi.nlm.nih.gov/), a database and retrieval system available from the National Center for Biotechnology Information $^{2}$ at the National Library of Medicine. The recently added structural dimension to Entrez, the Molecular Modeling Database (MMDB)--designed by project director Steve Bryant, along with postdocs Hitomi Ohkawa and Chris Hoguewill make it more useful to the structural community. Entrez enables a researcher to search relevant literature abstracts, protein or DNA sequences, or three-dimensional structures, drawn from various available databases (for example, Swiss-Prot or the PDB) and all linked so that a user can easily access a protein sequence, a corresponding structure (if available) and relevant references. Besides finding a document by 


\section{editorial}

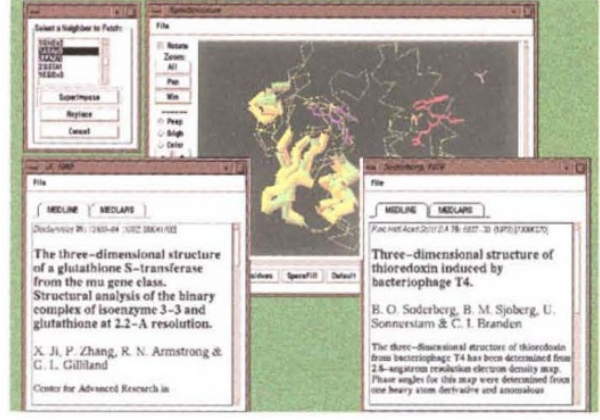

query, Entrez finds 'neighbours' of any document under current view; one may, for example, retrieve a protein sequence that has no available corresponding structure, then look for sequence neighbours that do have a corresponding structure in hopes of gaining structural insight. One may also spatially map residues of the structurally unsolved protein onto the structure of its sequence neighbour, useful for designing experiments. Structural neighbouring through MMDB will be available by March 15, according to Bryant, as well as a native-client structure viewer allowing graphical superposition of images. Searches for structural neighbours on the fly' using coordinates provided by the user-rather than from the A structural biologist's view of Entrez.

MMDB list of pre-compiled matches--is a longer-term goal.

There are, of course, other useful structural databases and research tools that are widely available; for example $\mathrm{SCOP}^{3}$ (Structural Classification of Proteins; http://scop.mrc-lmb.cam.ac.uk/scop/), which organizes protein structures hierarchically, identifying likely evolutionary relationships. In providing structural information that is already organized at a high level (beyond mere similarity), SCOP complements information on structural similarity provided by other methods. The structural homology search program Dali ${ }^{4}$ (www.embl-heidelberg.de/dali/dali.html) is another example; space constraints prevent giving a comprehensive list of useful structural research tools here. It is encouraging to see the degree of cooperation that exists between the creators of the various applications. For example, the designers of MMDB and SCOP cross-validate the structural similarities they find. Further, SCOP will start using MMDBs similarity scores. And most of the structural database or research tool web sites have links to many of the others.

As the implementation of databases and research tools improves, more and more of us will depend on them to an ever greater degree. Because of this, there should be a consensus within the structural community about standards for their reliability. As with any scientific information available only on the Internet-often by nature inappropriate for a paper journal by virtue of size, searchability or connectivity-issues of proper attribution and peer review arise. Although most of these problems are easily foreseen (though not always easily dealt with) some are not readily apparent. For example, when reviewers request that an author include for consideration in a paper information on a structural similarity that has not been published, the author can counter that the information has not been peer reviewed. But what if the information exists in an unreviewed database? Should the author be compelled to consider it? Often, only portions (algorithms, general descriptions) of databases are reviewed; how will this hypothetical dispute be best resolved in such a case? As applications get more sophisticated, this problem will intensify. Perhaps there should be some mechanism to review databases in their entirety, in situ on the Internet.

As DNA sequences first became widely available, scientists could publish findings of sequence homology in the most prestigious journals. Now that it is standard practice to search for sequence homology to a new gene, the number of such papers in top journals has declined, essentially in inverse proportion to the number of homologies discovered. It will be some time before stand-alone papers describing significant structural similarities lose their interest, though, especially if the comparison allows reasonable evolutionary or functional conclusions to be drawn. This may be partly because structures are intrinsically more information-laden than sequences. However, as information technology progresses along with the field, it may be expected that all but the most striking structural comparisons will loose some of their impact because they have become so routine.

1. Holm, L. \& Sander, C. Proteins Struct. Funct. Genet. 19, 165-173 (1994).

2. Schuler, G.D., Epstein, J.A. Ohkawa, H. \& Kans, J.A. Meths Enzymol. in the press.

3. Murzin, A.G., Brenner, S.E., Hubbard, T. \& Chothia, C. J. Mol. Biol. 247, 536-540 (1995).

4. Holm, L. \& Sander, C. J. Mol. Biol. 223, 123-138 (1993). 